\title{
Profilaxis antimicrobiana y complicaciones en apendicitis no complicada en un Hospital Nacional
}

\author{
Antimicrobial prophylaxis and complications in uncomplicated appendicitis in a \\ National Hospital
}

\author{
Gustavo, Bastidas P. y'Milton A., Tello C. ${ }^{(1)}$ \\ ${ }^{(1)}$ Facultad Medicina Humana, Universidad Nacional del Centro del Perú \\ Email: gubapa@hotmail.com
}

\section{Resumen}

Objetivo: Comparar el porcentaje de complicaciones post operatorias y la estancia hospitalaria en pacientes operados por apendicectomía convencional que recibieron tratamiento antimicrobiano profiláctico y tratamiento antimicrobiano terapéutico. en el Hospital Nacional Ramiro Prialé Prialé periodo abril-julio del 2014.

Material y métodos: Se diseñó un estudio prospectivo, observacional, transversal y analítico. La población estuvo conformada por los pacientes operados de apendicectomía convencional con diagnóstico de apendicitis aguda no complicada en el Servicio de Emergencia. La investigación se realizó con toda la población según criterios de inclusión y exclusión. El número total fue de 90 pacientes. La técnica de recolección de datos fue la encuesta y el instrumento un Formulario ad hoc.

Resultados: Existen iguales complicaciones post operatorias en el grupo con tratamiento antimicrobiano profiláctico y tratamiento antimicrobiano terapéutico; asi mismo, existen diferencias en el tiempo de estancia hospitalaria de los grupos de comparación.

Conclusiones: No existe diferencia significativa en el porcentaje de complicaciones post operatorias en pacientes que recibieron tratamiento antimicrobiano profiláctico comparado con los que recibieron tratamiento antimicrobiano terapéutico $(p=0.24)$ y existe diferencia significativa en la estancia hospitalaria de pacientes que recibieron tratamiento antimicrobiano profiláctico comparado con los que recibieron tratamiento antimicrobiano terapéutico $(p=0.005)$.

Palabras claves: drenaje quirúrgico, complicación quirúrgica, apendicitis aguda

\begin{abstract}
Objective: To compare the percentage of post-operative complications and hospital stay in patients undergoing conventional appendectomy who received prophylactic antimicrobial therapy and therapeutic antimicrobial treatment in the Hospital Nacional Ramiro Prialé Prialé during april to july 2014.

Material and methods: A prospective, observational, cross-sectional analytical study was designed. The population consisted of patients undergoing conventional appendectomy with acute uncomplicated appendicitis in the emergency department. The research was conducted with the entire population according to criteria of inclusion and exclusion. The total number was 90 patients. The data collection technique was the survey instrument and an ad hoc form .

Results: There are equal postoperative complications in the group with prophylactic antimicrobial therapy and therapeutic antimicrobial treatment. There is a difference in the length of hospital stay of the comparison groups.

Conclusions: There is no significant difference in the percentage of postoperative complications in patients receiving prophylactic antimicrobial therapy compared with those receiving therapeutic antimicrobial treatment $(p=0.24)$ and there is significant difference in hospital stay of patients receiving prophylactic antimicrobial therapy compared with receiving the therapeutic antimicrobial treatment $(p=0.005)$.
\end{abstract}

Keywords: surgical drainage, surgical complications, acute appendicitis 


\section{Introducción}

La apendicitis aguda es la causa más común de emergencia quirúrgica no traumática. ${ }^{(1)}$ El Hospital Nacional Ramiro Prialé Prialé EsSalud tuvo 370 casos de apendicitis aguda el $2013^{(2)}$. La Infección de Sitio Operatorio (ISO) es la complicación post operatoria más común y representa una amenaza significativa en términos de morbilidad, mortalidad y costos en los servicios de salud en el mundo entero. ISO es la segunda causa de infección intra hospitalaria representando aproximadamente un cuarto de los dos millones de casos de infecciones intrahospitalarias en los Estados Unidos de America. $^{(3)(4)}$ Entre un estimado de 27 millones de procedimientos quirúrgicos, ISO son reportados en cerca de 500,000 casos cada año. Se ha estimado que entre el $2 \%$ y $5 \%$ de pacientes sometidos a procedimientos extra abdominales limpios y hasta $20 \%$ de pacientes sometidos a procedimientos abdominales desarrollan ISO. ${ }^{(5)(10)}$

La profilaxis antimicrobiana (PA) apropiada ha demostrado ser efectiva en reducir la incidencia de ISO. La selección del agente antimicrobiano adecuado depende del patógeno más frecuente en causar la infección. Una simple dosis de antimicrobiano pre operatorio es suficiente para operaciones que duran hasta cuatro horas $^{(11)}$. Sin embargo en cirugías más prolongadas se necesitan dosis adicionales para mantener los niveles del medicamento. Se debe considerar una nueva administración en casos de prolongación de la cirugía o excesivo sangrado. El momento de la profilaxis antimicrobiana se considera óptima si la administración se da 30 minutos antes de la incisión ${ }^{(12)}$.

La información publicada refiere que entre el $30 \%$ y $50 \%$ de antimicrobianos son utilizados en los hospitales para PA y que el $30 \%$ a $90 \%$ de esta profilaxis es inapropiado. Los agentes antimicrobianos son sobre prescritos, aplicados en un periodo inadecuado y continuados por largos periodos ${ }^{(13)}$. Un estudio en la india describe que aproximadamente 55\% de los cirujanos prescriben un único antimicrobiano para cirugías limpias. Una combinación de dos o más agentes anti microbianos se prefieren en cirugías limpias contaminadas $42.3 \%$ y $46.9 \%$ en cirugías sucias. Las cefalosporinas de tercera generación, particularmente ceftriaxona y cefotaxima, fueron los antimicrobianos más frecuentemente utilizados $80 \%$ por los cirujanos en todos los procedimientos.

Sin embargo, en la mayoría de los casos, los antimicrobianos son utilizados por periodos prolongados mayores a los referidos en las guías clínicas ${ }^{(4)}$. El uso inapropiado de antimicrobianos está asociado con incrementos innecesarios en los costos de tratamiento y en el aumento de la resistencia antimicrobiana ${ }^{(15)}$. En el Perú debido a una falta de información adecuada y guas clínicas para profilaxis antimicrobiana en cirugía abdominal hay una necesidad de generar una línea de base en el patrón de uso de PA. El objetivo de la investigación es comparar el porcentaje de complicaciones post operatorias entre paciente operados por apendicitis aguda no complicada con profilaxis antimicrobiana y terapia antimicrobiana y su estancia hospitalaria.

\section{Materiales y métodos:}

Método científico: el método que se usará para resolver el problema será el método prolectivo.

Tipo de investigación: estudio prospectivo, observacional, transversal y analítico.

Población o universo: pacientes operados de apendicectomía convencional con diagnóstico de apendicitis aguda no complicada en el Servicio de Emergencia del Hospital Nacional Ramiro Prialé Prialé EsSalud Huancayo.

Muestra: no probabilística.

Técnicas de recolección de datos: la técnica que se siguió en la presente investigación fue la encuesta a las Historias Clínicas de los pacientes con criterios de inclusión y exclusión. La aplicación del instrumento se llevó a cabo entre los meses de Abril a Julio de 2014 de acuerdo al cronograma de la investigación.

Procesamiento de datos: para el análisis estadístico se utilizó el paquete estadístico IBM SPSS Statistics versión 21.

Procedimiento a seguir para probar las hipótesis: las variables categóricas se describieron mediante frecuencias y porcentajes como medidas de tendencia central y con Intervalos de confianza al 95\%. Las variables numéricas se describieron mediante la media, o mediana como medida de tendencia central y la desviación típica, o rangos intercuartiles como medida de dispersión. La comparación de proporciones y medias se realizaron con pruebas paramétricas y no paramétricas.

\section{Resultados}

Tabla I.

Características de los pacientes apendicectomizados por emergencia en el hospital nacional ramiro Prialé Prialé EsSalud Huancayo

\begin{tabular}{|l|c|c|c|}
\hline & $\begin{array}{c}\text { Con Profilaxis } \\
(\mathrm{n}=40)\end{array}$ & $\begin{array}{c}\text { Con Tratamiento } \\
(\mathrm{n}=50)\end{array}$ & $p$ Valor \\
\hline Edad (años)† & $22.4 \pm 16.3$ & $23.2 \pm 11.1$ & $0.07^{* *}$ \\
\hline Sexo (M:F) & $28: 12$ & $33: 17$ & $0.06^{*}$ \\
\hline Peso (kg) & $62.5 \pm 8.8$ & $63.2 \pm 10.4$ & $0.73^{* *}$ \\
\hline Goldman (I:II:III) & $0: 35: 5$ & $2: 43: 5$ & $0.81^{*}$ \\
\hline "Prueba de Mann Whitney; ${ }^{* *}$ Prueba de $x^{2}$ \\
\hline †Media \pm DE
\end{tabular}

En la Tabla I, se comparan en los dos grupos las siguientes variables: edad, sexo, peso y riesgo pre quirúrgico Goldman. El riesgo quirúrgico más frecuente fue el Goldman II. 
Tabla 2.

Tipo de terapia antimicrobiana y estancia hospitalaria en pacientes apendicectomizados por emergencia en el hospital nacional Ramiro Prialé Prialé EsSalud Huancayo 2014

\begin{tabular}{|c|c|c|c|}
\hline & $\begin{array}{l}\text { Con Profilaxis } \\
\quad(n=40)\end{array}$ & $\begin{array}{c}\text { Con Tratamiento } \\
(n=50)\end{array}$ & $p$ Valor \\
\hline $\begin{array}{l}\text { Diámetro máximo } \\
\text { del apéndice cecal } \dagger\end{array}$ & $11.6 \pm 2.5$ & $12.3 \pm 2.8$ & $0.14^{* * *}$ \\
\hline Tiempo operatorio† & $47.8 \pm 8.6$ & $55.4 \pm 10.9$ & $0.08^{* * *}$ \\
\hline $\begin{array}{l}\text { Complicaciones } \\
\text { post operatorias }\end{array}$ & $11: 29$ & $9: 41$ & $0.24^{* *}$ \\
\hline \begin{tabular}{|l|}
$\begin{array}{l}\text { ISO incisional } \\
\text { superficial }\end{array}$ \\
\end{tabular} & $2: 38$ & $2: 48$ & $0.22^{* *}$ \\
\hline $\begin{array}{l}\text { ISO incisional } \\
\text { profundo }\end{array}$ & $3: 37$ & $2: 48$ & $0.10^{*}$ \\
\hline $\begin{array}{l}\text { ISO espacio } \\
\text { órgano }\end{array}$ & $1: 39$ & $1: 49$ & $0.08^{*}$ \\
\hline Fistula cecal & $2: 38$ & $1: 49$ & $0.07^{*}$ \\
\hline ITU & $2: 38$ & $2: 48$ & $0.06^{*}$ \\
\hline $\begin{array}{l}\text { Neumonía intra } \\
\text { hospitalaria }\end{array}$ & $1: 39$ & $1: 49$ & $0.09^{*}$ \\
\hline
\end{tabular}

En la Tabla 2, se aprecian los hallazgos y las complicaciones post operatorias de los pacientes operados de apendicitis aguda por emergencia con y sin la utilización de drenaje quirúrgico. La mayor complicación fue la ISO incisional profundo.

Tabla $\mathbf{N}^{\circ} \mathbf{3}$ Tipo de terapia antimicrobiana y estancia hospitalaria en pacientes apendicectomizados por emergencia en el hospital nacional Ramiro Prialé Prialé EsSslud Huancayo 2014

\begin{tabular}{|l|c|c|c|}
\hline & $\begin{array}{c}\text { Con Profilaxis } \\
(n=16)\end{array}$ & $\begin{array}{c}\text { Con Tratamiento } \\
(n=82)\end{array}$ & $p$ Valor \\
\hline $\begin{array}{l}\text { Estancia } \\
\text { hospitalaria } †\end{array}$ & $2.69 \pm 1.23$ & $3.78 \pm 1.67$ & $0.005^{*}$ \\
\hline *Prueba de Mann Whitney & \multicolumn{3}{|l}{} \\
\hline \multicolumn{2}{|l}{ †Media \pm DE }
\end{tabular}

En la Tabla 3, se observa la comparación de la estancia hospitalaria entre pacientes apendicectomizados por Emergencia con y sin profilaxis antimicrobiana.

\section{Discusión}

Los grupos de comparación muestran que no hay diferencia significativa en ninguna de sus características generales. Las complicaciones post operatorias no tuvieron diferencia significativa entre los dos grupos de comparación $(p=0.24)$. Tampoco se determinaron diferencias significativas en las variables diámetro máximo $(p=0.14)$ y tiempo operatorio $(p=0.08)$. Estos resultados son compatibles con las investigaciones realizadas por Bauer y $y^{(160) . ~(1989), ~ S o n g ~(172 o l . ~(1998), ~ A n d e r s e n ~}$ y col'(2003), Kasatpibal y ('21). (2006), Ravari y y (201 I), Choi (21) col. (201 I), Rajabi-Mashadi ${ }^{(22)}$ y col. (2012) y Hussain ${ }^{(23)}$ y col. $(2012)$.

A pesar de los diversos esquemas utilizados en la profilaxis antimicrobiana en las diferentes investigaciones, los resultados determinan que no hay ventaja en el uso de tratamiento antimicrobiano en pacientes opera- dos de apendicectomía convencional por apendicitis no complicada. Estas semejanzas se pueden explicar por el espectro antimicrobiano de los medicamentos utilizados. Todos los esquemas empleados tienen espectro contra bacterias gram negatilivas y anaerobios.

Andersen y col. (2010) determinaron que la profilaxis tiene iguales resultados cuando se aplica en el pre, intra o post operatorio. La aplicación de la profilaxis antimicrobiana en nuestra investigación se dio en el momento del llamado telefónico del personal de enfermería de sala de operaciones al personal de enfermería del servicio de emergencia solicitando que el paciente sea llevado al centro quirúrg(č para ser operado.

Rajabi-Mashadi y col. (2012) y Hussain y col. (2012) determinaron que la prolongación del tratamiento antimicrobiano no tiene ninguna ventaja en relación con la profilaxis antimicrobiana. En nuestra investigación se aplicó una sola dosis de cefazolina de un gr. por vía endovenosa y metronidazol 500 mg. por vía endovenosa 
al llamado de centro quirúrgico. Estos hallazgos sugieren la relación entre el tiempo operatorio, que para ambos grupos fue inferior a sesenta minutos, con el tiempo de vida media de los antimicrobianos utilizados.

Además estos resultados tienen relación con la estancia hospitalaria de los grupos comparados donde se encontró una diferencia significativa entre ambos $(p=0.005)$. El desarrollo de complicaciones en el post operatorio en pacientes con diagnóstico de apendicitis aguda no complicada está relacionado con diversos factores entre los cuales se puede mencionar el tiempo de estancia hospitalaria en el post operatorio y el estado nutricional del paciente.

Se debe resaltar que no existen investigaciones que hayan comparado la estancia hospitalaria de pacientes operados de apendicectomía convencional con diagnóstico de apendicitis aguda no complicada en nuestro país por lo que la presente es la primera de este tipo que permitirá la mejor toma de decisiones para la atención de los pacientes.

\section{Conclusiones}

No existe diferencia significativa en el porcentaje de complicaciones post operatorias en pacientes que recibieron tratamiento antimicrobiano profiláctico comparado con los que recibieron tratamiento antimicrobiano terapéutico $(p=0.24)$.

Existe diferencia significativa en la estancia hospitalaria de pacientes que recibieron tratamiento antimicrobiano profiláctico comparado con los que recibieron tratamiento antimicrobiano terapéutico $(p=0.005)$.

\section{Recomendaciones}

Se recomienda al Hospital Nacional Ramiro Prialé Prialé a través del Jefe de Servicio de Cirugía General considerar en su Guía de Practica Clínica para Apendicitis Aguda No Complicada la indicación del uso de la antimicrobiano profilaxis con cefazolina y metronidazol.

Se recomienda al Hospital Nacional Ramiro Prialé Prialé a través del Jefe de Servicio de Cirugía General que se propicie el alta precoz a los pacientes con diagnóstico de apendicitis aguda no complicada.

\section{Referencias}

Vásquez V. Abdomen agudo quirúrgico. Revista de la Facultad de Medicina Humana. 1999; I ( ): 29-33.

Servicio de Anatomía Patológica del Hospital Nacional Ramiro Prialé Prialé. 2013.

Martone WJ, Nochols RL. Recognition, prevention, sur- veillance and management of surgical site infections. Introduction to the problem and symposium overview. Clin Infect Dis 200I; 33 Suppl 2: S67-S68.

Haley RW, Culver DH, White JW, Morgan WM, Emori TG. The nationwide nosocomial infection rate: a new need for vital statistics. Am J Epidemiol 1985; 121 : 159- 167.

Delgado Rodríguez M, Sillero Arenas M, Medina Cuadros M, Martinez Gallego G. Nosocomial infections in surgical patients: comparison of two measures of intrinsic patient risk. Infect Control Hosp Epidemiol 1997; 18(1): 19-23.

Cardo D, Horan T, Andrus M, Dembinski M, Edwards J. National Nosocomial Infections Surveillance (NNIS) System Report, data summary from January 1992 through June 2004. Am J Infect Control 2004; 32(8): 470-85.

Mangram AJ, Horan TC, Pearson ML, Silver LC, Jarvis WR. Guide for Prevention of Surgical Site Infection. Infect Control Hosp Epidemiol. 1999; 20(4): 247278.

Horan TC, Emori TG. Definitions of key terms used in the NNIS System. Am J Infect Control 1997; 25(2): $112-1 \mid 6$.

Wallace WC, Cinat M, Gornick WB, Lekawa ME, Wilson SE. Nosocomial infections in the surgical intensive care unit: a difference between trauma and surgical patients. Am Surg 1999; 65(10): 987-990.

Scheel O, Stormark M. National prevalence survey on hospital infections in Norway. J Hosp Infect 1999; 4l (4): 33 I-335.

McDonald M, Grabsch E, Marshall C, Forbes A. Single versus multiple-dose antimicrobial prophylaxis for major surgery: a systematic review. Aust N Z J Surg 1998; 68(6): 388-396.

Scottish Intercollegiate Guidelines Network. Antibiotic prophylaxis in surgery. Available from URL: http:// www.sign.ac.uk/pdf/sign I04.pdf [cited 2009 Mar 5].

Dettenkofer M, Forster DH, Ebner W, Gastmeier P, Ruden $\mathrm{H}$, Daschner FD. The practice of perioperative antibiotic prophylaxis in eight German hospitals. Infection 2002; 30(3): 164-167.

Hya SS, Thatte UM. Patterns of antimicrobial use by surgeons in India. Ind J Surg 2005; 67(6): 308-3I 5.

Nichols RL. Preventing surgical site infections. Clin Med Res. 2004; 2(2): 1 15-1 18. 
Bauer T, Vennitis B, Holm B, Hahn-Pedersen J. Antibiotic prophilaxis in acute nonperforated appendicitis. Ann Surg. 1989; 209(3): 307-3।I.

Song F, Glenny AM. Antimicrobial prophylaxis in colorectal surgery: a systematic review of randomized controlled trials. Br J Surg. 1998; 85(9): I232- I24I.

Andersen BR, Kallehave FL, Andersen HK. Antibiotics versus placebo for prevention of postoperative infection after appendicectomy. Cochrane Database Syst Rev. 2003; (2):CD00 I 439.

Kasatpibal N, Nørgaard M, Sørensen HT, Schønheyder $\mathrm{HC}$, Jamulitrat S. Risk of surgical site infection and efficacy of antibiotic prophylaxis: a cohort study of appendectomy patients in Thailand. BMC Inf Dis. 2006; III(6): I-7.

Ravari H, Jangjoo A, Motamedifar J, Moazzami K. Oral metronidazole as antibiotic prophylaxis for patients with nonperforated appendicitis. Clin Exp Gastroenterol. 201 I;4: 273-276.

Choi J], Ranasinghe NE, Lynn ET, Divino CM .Postoperative antibiotics correlate with worse outcomes after appendectomy for nonperforated appendicitis. I Am Coll Surg. 201 I; 213(6): 778-783.

Rajabi-Mashadi MT, Mousavi SH, Khosravi-Mashizi MH, Ghayour-Mobarhan M, Sahebkar A. Optimum duration of perioperative antibiotic therapy in patients with acute non-perforated appendicitis: a prospective randomized trial. Asian Biomedicine. 2012; 6(6): 891-894

Hussain MI, Alam MK, Al-Qahatani HH, Al-Akeely MH. Role of postoperative antibiotics after appendectomy in non-perforated appendicitis. J Coll Physicians Surg Pak. 20 I2; 22(I2): 756-759.

Kyriazis AA, Esterly JR. Development of lymphoid tissues in the human embryo and early fetus. Arch Pathol. 1970; 90(4):348-353.

Schumpelick V, Dreuw B, Ophoff K, Preschr A. Appendix and Cecum, Embryology, Anatomy, and Surgical Applications. Surgical Clinics of North America. 2000; 80(I): 295-3I8.

Klingler PJ, Seelig MH, DeVault KR. Ingested foreign bodies within the appendix: A 100-year review of the literature. Dig Dis. 1998; 16(5): 308-3।4.

Carr NJ. The pathology of acute appendicitis. Ann Diag Pathol. 2000; 4(I):46-58.
Birnbaum BA, Wilson SR. Appendicitis at the millennium. Radiology. 2000; 215(2): 337-348.

Dominguez EP, Sweeney JF, Choi YU. Diagnosis and Management of Diverticulitis and Appendicitis. Gastroenterol Clin N Am. 2006; 35(2): 367-39l.

Andersen BR, Kallehave FL, Andersen HK. Antibiotics versus placebo for prevention of postoperative infection after appendicectomy. Cochrane Database Syst Rev. 2005; 3: CD00 I 439.

Le D, Rusin W, Hill B, Langell J. Post-operative antibiotic use in nonperforated appendicitis. The American Journal of Surgery. 2009; 198(6), 748-752

Wright JE. Controlled trial of wound infiltration with bupivicaine for postoperative pain relief after appendectomy in children. Br J Surg. 1993; 80( I), I I0-1 I I. 